\title{
Coupling between M2-branes and Form Fields
}

\author{
Yoonbai Kim, O-Kab Kwon, Hiroaki Nakajima, D. D. Tolla ${ }^{1}$ \\ Department of Physics, BK21 Physics Research Division, Institute of Basic Science, \\ ${ }^{1}$ University College \\ Sungkyunkwan University, Suwon 440-746, Korea \\ yoonbai, okab, nakajima, ddtolla@skku.edu
}

\begin{abstract}
In the context of low-energy effective theory of multiple M2-branes, we construct the interaction terms between the world-volume fields of M2-branes and the antisymmetric tensor fields of three- and six-forms. By utilizing the compactification procedure, we show coincidence between the dimensionally reduced coupling and the R-R coupling to D-branes in type II string theory. We also discuss that a cubic term proportional to six-form field reproduces the quartic mass-deformation term in the world-volume theory of multiple M2-branes.
\end{abstract}




\section{Introduction}

To the leading order, the low-energy dynamics of a stack of $N$ parallel D-branes is described by the super Yang-Mills action with $\mathrm{U}(N)$ gauge symmetry and the couplings to the bulk fields. In string theory, D-branes carry R-R charges and couple to R-R fields. The form of interaction is given by Wess-Zumino(WZ)-type action [1, 2, 3]. Unlike the case of a single D p-brane where it couples only to R-R fields of rank $p+1$ or less, a stack of $N$ parallel D-branes couples to all even-form R-R fields in type IIB string theory and to all odd-form R-R fields in type IIA string theory.

Analogous to the D-branes of string theory, in M-theory, we have the M2- and M5-branes and the corresponding three- and six-form fields. About their dynamics, the construction of the world-volume action of multiple M-branes as well as their coupling to the form fields is more difficult than that of multiple D-branes. Recently the world-volume description of low-energy dynamics of multiple M2-branes is available, which is Bagger-Lambert-Gustavsson (BLG) theory with $\mathcal{N}=8$ supersymmetry and $\mathrm{SU}(2) \times \mathrm{SU}(2)$ gauge symmetry [4, 5] and Aharony-BergmanJafferis-Maldacena (ABJM) theory with $\mathcal{N}=6$ manifest supersymmetry and $\mathrm{U}(N) \times \mathrm{U}(N)$ (or $\mathrm{SU}(N) \times \mathrm{SU}(N)$ gauge symmetry) [6]. Once the action of world-volume fields is obtained, reproduction of its string theory limit is an attractive research direction. The reduction to type IIA string theory of the BLG theory upon circle compactification in the direction transverse to the M2-brane has been achieved in Ref. [7, 8].

Despite an overwhelming progress in the understanding of the world-volume action of multiple M2-branes, a little have been done to couple them to the bulk form fields [9, 10]. Therefore, it is intriguing to construct the mutual interaction between the three- or six-form fields and the world-volume fields in the context of BLG and ABJM theories. It is the main objective of this paper to make a proposal for the action which describes the interaction between the M2-branes and the form fields of arbitrary transverse field dependence in the context of BLG theory and to verify the proposal by reducing it to a similar interaction term in type IIA string theory through circle compactification.

To see the importance of these interaction terms, we make a quick comparison between M2brane dynamics and the corresponding D2-brane in string theory. In analogy with D2-brane dynamics, in the presence of nonvanishing three-form and dual six-form fields, the low energy dynamics of multiple parallel M2-branes is expected to be described by both their world-volume action, the BLG action $S_{\mathrm{BLG}}$ in our case, and the coupling between M2-branes and form fields $S_{C}$,

$$
S_{11}=S_{\mathrm{BLG}}+S_{C}
$$

On the other hand, in the type IIA superstring theory the D2-brane action possesses the Dirac- 
Born-Infeld (DBI) type (or Yang-Mills type in low energy limit) world-volume action of $N$ parallel D2-branes $S_{\mathrm{DBI}}$ and the R-R coupling $S_{\tilde{C}}$,

$$
S_{10}=S_{\mathrm{DBI}}+S_{\tilde{C}}
$$

In nontrivial background, the DBI action is given in terms of the gauge-invariant field strength $\tilde{F}_{\mu \nu}+\tilde{B}_{\mu \nu}$, where $\tilde{F}_{\mu \nu}$ is the field strength of the $\mathrm{U}(N)$ gauge field and $\tilde{B}_{\mu \nu}$ is the NS-NS two-form field. In the absence of $S_{C}$, it have been verified that, upon circle compactification, the action $S_{\text {BLG }}$ reduces to Yang-Mills matter action composed of only $\tilde{F}_{\mu \nu}$. In this paper we show that, after the compactification, the presence of $S_{C}$ not only produces $S_{\tilde{C}}$ it also gives the missing $\tilde{B}_{\mu \nu}$ piece of the DBI action in the NS-NS background.

Some particular configuration of form fields coupled to D-branes or M-branes can be regarded as the mass deformation of world-volume theories [11, 12]. The (SUSY-preserving) mass deformation of BLG theory is explicitly constructed in [13, 14], which contains the quartic coupling among scalar fields as well as the quadratic mass terms. We show that the WZ-type coupling with particular configuration of form fields reproduces this quartic coupling.

The remaining part of this paper is organized as follows. In section 2 we put forward our proposal for the three- and six-form couplings to multiple M2-branes in BLG theory. Our proposal is made in parallel with the known multiple D2-brane coupling to R-R forms. In section 3 we show that the circle compactification of the action reproduces the corresponding action in tendimensional IIA string theory. In section 4 we single out a particular term in the six-form coupling and show that, with a proper choice of the constant background form field, it gives rise to the quartic mass deformation of the BLG theory. Section 5 is devoted to conclusions and discussions.

\section{Coupling between M2-branes and Form Fields in BLG Theory}

In string theory the coupling of any D $p$-brane to R-R form fields $\tilde{C}_{(n)}$ is given by the WZ-type action as [1, 2, 3]

$$
S_{\tilde{C}}=\mu_{p} \int_{p+1} \operatorname{STr}\left(P\left[e^{i \tilde{\lambda} \tilde{X}_{\tilde{X}} \tilde{X}} \sum \tilde{C}_{(n)} e^{\tilde{B}}\right] e^{\tilde{\lambda} \tilde{F}}\right)
$$

where $\mu_{p}$ is R-R charge of the $\mathrm{D} p$-brane, $\tilde{\lambda}=2 \pi l_{\mathrm{s}}^{2}$ is the string scale, $\tilde{X}$ is the transverse scalar field, $\tilde{B}$ is the NS-NS two-form field, and $\tilde{F}=d \tilde{A}$ is field strength of the gauge field $\tilde{A}$. The trace

\footnotetext{
${ }^{1}$ We put tildes for the fields and the parameters in string theory.
} 
is taken over the gauge indices, $P[\ldots]$ denotes pullback, the summation is taken over all the R-R forms, and $i_{\Phi}$ represents an interior product by $\Phi^{i}$, explicitly written

$$
\mathrm{i}_{\Phi} \tilde{C}^{(n)}=\frac{1}{(n-1) !} \Phi^{i} \tilde{C}_{i i_{1} \ldots i_{n-1}} d x^{i_{1}} \wedge \ldots \wedge d x^{i_{n-1}}
$$

For later convenience we expand the exponential $e^{i \tilde{\lambda} \mathrm{i}} \tilde{\mathrm{x}} \tilde{\mathrm{x}}$ and write explicitly the first few terms in the case of $p=2$,

$$
\begin{aligned}
S_{\tilde{C}}=\mu_{2} \int & \frac{1}{3 !} d x^{\mu} \wedge d x^{\nu} \wedge d x^{\rho} \operatorname{STr}\left\{\tilde{C}_{\mu \nu \rho}+3 \lambda \tilde{C}_{\mu \nu i} \tilde{D}_{\rho} \tilde{X}_{i}+3 \lambda^{2} \tilde{C}_{\mu i j} \tilde{D}_{\nu} \tilde{X}_{i} \tilde{D}_{\rho} \tilde{X}_{j}\right. \\
& +\lambda^{3} \tilde{C}_{i j k} \tilde{D}_{\mu} \tilde{X}_{i} \tilde{D}_{\nu} \tilde{X}_{j} \tilde{D}_{\rho} \tilde{X}_{k}-3 i \lambda^{2} \tilde{C}_{\mu i j} \tilde{X}_{i} \tilde{X}_{j} F_{\nu \rho}-3 i \lambda^{3} \tilde{C}_{i j k} \tilde{X}_{i} \tilde{X}_{j} \tilde{D}_{\mu} \tilde{X}_{k} F_{\nu \rho} \\
& -i \lambda \tilde{C}_{\mu \nu \rho i j} \tilde{X}_{i} \tilde{X}_{j}-3 i \lambda^{2} \tilde{C}_{\mu \nu i j k} \tilde{X}_{i} \tilde{X}_{j} \tilde{D}_{\rho} \tilde{X}_{k}-3 i \lambda^{3} \tilde{C}_{\mu i j k l} \tilde{X}_{i} \tilde{X}_{j} \tilde{D}_{\nu} \tilde{X}_{k} \tilde{D}_{\rho} \tilde{X}_{l} \\
& -i \lambda^{4} \tilde{C}_{i j k l m} \tilde{X}_{i} \tilde{X}_{j} \tilde{D}_{\mu} \tilde{X}_{k} \tilde{D}_{\nu} \tilde{X}_{l} \tilde{D}_{\rho} \tilde{X}_{m}-\frac{3}{2} \lambda^{3} \tilde{C}_{\mu i j k l} \tilde{X}_{i} \tilde{X}_{j} \tilde{X}_{k} \tilde{X}_{l} F_{\nu \rho} \\
& \left.-\frac{3}{2} \lambda^{4} \tilde{C}_{i j k l m} \tilde{X}_{i} \tilde{X}_{j} \tilde{X}_{k} \tilde{X}_{l} \tilde{D}_{\mu} \tilde{X}_{m} F_{\nu \rho}+\cdots\right\}
\end{aligned}
$$

where $\tilde{X}_{i}(i=1,2, \ldots, 7)$ are seven transverse adjoint scalar fields with $\tilde{D}_{\mu} \tilde{X}_{i}=\partial_{\mu} \tilde{X}_{i}+i\left[A_{\mu}, \tilde{X}_{i}\right]$. Here we omitted the $\tilde{C} \wedge \tilde{B}$-terms for simplicity. In M-theory we naturally expect a similar coupling between the M-branes (M2- and M5-branes) and the antisymmetric form fields (the three-form field $C_{(3)}$ and the dual six-form field $\left.C_{(6)}\right)$ [15]. Since $\tilde{B}$ is already a part of $C_{(3)}, C_{8 i j} \sim \tilde{B}_{i j}$ for the compactified eighth direction, the expected action involves the interaction between the form fields and the world-volume fields. In the following, we will consider the BLG theory and construct an analogue of the coupling between the world-volume fields and the form fields. Instead of the original formulation based on three-algebra, we employ a familiar gauge theory formulation [16, 17].

We begin with the BLG theory with eight transverse bi-fundamental scalar fields $X_{I}(I=$ $1,2, \ldots, 8)$ and two gauge fields $A$ and $\hat{A}$ of $\mathrm{SU}(2) \times \mathrm{SU}(2)$ gauge symmetry. The bosonic part of the action is

$$
S_{\mathrm{bos}}=S_{X}+S_{\mathrm{CS}}+S_{C}
$$

The first two are well established and are given by

$$
\begin{aligned}
S_{X} & =\int d^{3} x \operatorname{Tr}\left[-\left(D_{\mu} X_{I}\right)^{\dagger} D^{\mu} X_{I}-\frac{32 \pi^{2}}{3 k^{2}} X_{I J K} X_{I J K}^{\dagger}\right], \\
S_{\mathrm{CS}} & =\frac{k}{4 \pi} \int d^{3} x \epsilon^{\mu \nu \rho} \operatorname{Tr}\left(A_{\mu} \partial_{\nu} A_{\rho}+\frac{2 i}{3} A_{\mu} A_{\nu} A_{\rho}-\hat{A}_{\mu} \partial_{\nu} \hat{A}_{\rho}-\frac{2 i}{3} \hat{A}_{\mu} \hat{A}_{\nu} \hat{A}_{\rho}\right),
\end{aligned}
$$

where $k$ is the Chern-Simons level and we have used the notation

$$
D_{\mu} X_{I}=\partial_{\mu} X_{I}+i A_{\mu}-i X_{I} \hat{A}_{\mu}, \quad X_{I J K} \equiv X_{[I} X_{J}^{\dagger} X_{K]}
$$


Due to T-duality between type IIA and IIB string theories, one can restrict possible interaction terms between D-branes and R-R form fields. The exponential factor of the gauge field $\tilde{F}$ in the R$\mathrm{R}$ coupling (2.1) is introduced along the line of open string tadpole computation and is compatible with the T-duality [1, 2, 18, 3. In addition gauge invariance on the D-brane requires $\tilde{B}+\tilde{\lambda} \tilde{F}$ combination and it justifies the exponential factor of the NS-NS two-form field in (2.1). Unlike the superstring theories, however, there seems no concrete guideline for the interactions between M-branes and form fields in M-theory yet. The candidate for the WZ-type coupling between M2-branes and form fields, which is linear in the form fields, is

$$
\begin{aligned}
S_{\tilde{C}}=\int_{2+1} \operatorname{Tr}\left(\mu_{2} P\left[C_{(3)}\right]+\right. & \left.\mu_{2}^{\prime} P\left[\left\langle\mathrm{i}_{X} \mathrm{i}_{X} \mathrm{i}_{X}\right\rangle C_{(6)}\right]\right) \\
=\int \frac{1}{3 !} d^{3} x \epsilon^{\mu \nu \rho} \operatorname{Tr}\left\{\frac{\mu_{2}}{2}[\right. & \frac{1}{2}\left(\hat{C}_{\mu \nu \rho}+C_{\mu \nu \rho}\right)+3 \lambda C_{\mu \nu I}\left(D_{\rho} X_{I}\right)^{\dagger} \\
& +\frac{3}{2} \lambda^{2}\left(\hat{C}_{\mu I J}\left(D_{\nu} X_{I}\right)^{\dagger} D_{\rho} X_{J}+C_{\mu I J} D_{\nu} X_{I}\left(D_{\rho} X_{J}\right)^{\dagger}\right) \\
& \left.+\lambda^{3} C_{I J K}\left(D_{\mu} X_{I}\right)^{\dagger} D_{\nu} X_{J}\left(D_{\rho} X_{K}\right)^{\dagger}\right]+(\text { c.c. }) \\
+\frac{\mu_{2}^{\prime}}{2}[ & C_{\mu \nu \rho I J K} X_{I J K}^{\dagger}+\frac{3}{2} \lambda \hat{C}_{\mu \nu I J K L}\left\langle\left\langle X_{I J K}^{\dagger} D_{\rho} X_{L}\right\rangle\right\rangle \\
& +\frac{3}{2} \lambda C_{\mu \nu I J K L}\left\langle\left\langle X_{I J K}\left(D_{\rho} X_{L}\right)^{\dagger}\right\rangle\right\rangle \\
& +3 \lambda^{2} C_{\mu I J K L M}\left\langle\left\langle X_{I J K}^{\dagger} D_{\nu} X_{L}\left(D_{\rho} X_{M}\right)^{\dagger}\right\rangle\right\rangle \\
& +\frac{1}{2} \lambda^{3} \hat{C}_{I J K L M N}\left\langle\left\langle X_{I J K}^{\dagger} D_{\mu} X_{L}\left(D_{\nu} X_{M}\right)^{\dagger} D_{\rho} X_{N}\right\rangle\right\rangle \\
& \left.+\frac{1}{2} \lambda^{3} C_{I J K L M N}\left\langle\left\langle X_{I J K}\left(D_{\mu} X_{L}\right)^{\dagger} D_{\nu} X_{M}\left(D_{\rho} X_{N}\right)^{\dagger}\right\rangle\right]+(\text { c.c. })\right\},
\end{aligned}
$$

where $\mu_{2}$ is M2-brane tension, $\lambda=2 \pi l_{\mathrm{P}}^{3 / 2}$ with Planck length $l_{\mathrm{P}}$, and $\mu_{2}^{\prime}=\beta \lambda \mu_{2}$. Dimensionless parameter $\beta$ will be fixed by requiring that when reduced to ten dimensions this action reproduces the correct D2-brane coupling to R-R and NS-NS form fields in type IIA superstring theory. $\left\langle\mathrm{i}_{X} \mathrm{i}_{X} \mathrm{i}_{X}\right\rangle$ denotes interior products by $X_{I J K}$ and its Hermitian conjugate $X_{I J K}^{\dagger}$ in gauge invariant manner. We introduce the notation $\langle\langle\ldots\rangle\rangle$ to symmetrize objects inside the trace, for instance,

$$
\begin{aligned}
& \left\langle\left\langle X_{I J K}^{\dagger} D_{\nu} X_{L}\left(D_{\rho} X\right)_{M}^{\dagger}\right\rangle\right\rangle \\
& \quad=\frac{1}{3}\left[X_{I J K}^{\dagger} D_{\nu} X_{L}\left(D_{\rho} X_{M}\right)^{\dagger}+\left(D_{\nu} X_{L}\right)^{\dagger} X_{I J K}\left(D_{\rho} X_{M}\right)^{\dagger}+\left(D_{\nu} X_{L}\right)^{\dagger} D_{\rho} X_{M} X_{I J K}^{\dagger}\right] .
\end{aligned}
$$

We also note that the different powers of the Planck length $l_{\mathrm{P}}$ in front of some of the terms in the action are chosen based on dimension counting and the $(2 \pi)^{n}$ factors are inserted to mimic the similar factors in ten dimensions. 
Now we recall that the scalar fields $X_{I}$ transform in the bi-fundamental representation of the gauge group $\mathrm{SU}(2) \times \mathrm{SU}(2)$, while $X_{I}^{\dagger}$ transform in the anti-bi-fundamental representation. In order to have gauge invariance, we realize that the $C_{\mu \nu \rho I J K}$ and all the other antisymmetric tensor fields with odd number of transverse indices should be in the bi-fundamental representation. For the same reason $C_{\mu \nu \rho}$ and all the other antisymmetric tensor fields $C$ with even number of

the transverse indices should be in the adjoint of the left $\mathrm{SU}(2)$, while $\hat{C}_{\mu \nu \rho}$ and all the other antisymmetric tensor fields $\hat{C}$ with even number of the transverse indices should be in the adjoint of the right $\mathrm{SU}(2)$. With these transformation rules all the terms in (2.9) are gauge invariant.

In Ref. [9], the authors proposed the WZ-type couplings in M-theory in terms of 3-algebras with Euclidean and Lorentzian metrics. Some of terms of their proposal for the WZ-type action resemble those in our action (2.3), however, in Ref. [9] the authors assumed that the three- and six-form fields do not depend on the transverse scalar fields and so transform trivially under the gauge transformation. To get a gauge invariant action they introduced symmetrized constant tensors originated from symmetrized trace of generators with 3-algebra indices. For a specific representation of the 3-algebra, the symmetrized tensors satisfying the gauge invariance of the action were obtained as functions of structure constant of the 3-algebra. After that, ten-dimensional WZ-type action was obtained by using the Higgs mechanism proposed in Ref. [7]. Since the resulting action is composed of constant form fields and symmetric tensors depending on specific representation of 3-algebra, it is not clear to relate the results to the known WZ-type action (2.1) expressed by $\mathrm{U}(2)$-adjoints.

\section{$3 \quad$ Reduction from M-theory to IIA String Theory}

In the previous section we constructed the analogue of WZ-type coupling (2.9) between M2-branes and form fields. In this section we shall test and justify the obtained candidate by comparing it with the R-R coupling in string theory (2.3) by reducing it to the ten-dimensional type IIA superstring theory. Specifically we expand the action (2.1) and compare the obtained result (2.3) with the dimensionally reduced WZ-type action of M-theory (2.9).

According to the compactification procedure of Ref. [7], we can split the transverse scalars into trace and traceless parts,

$$
\begin{aligned}
X_{i} & =\check{x}_{i}+i \mathbf{x}_{i}, \quad(i=1,2, \ldots, 7), \\
X_{8} & =\frac{v}{2} \mathbf{1}+\check{x}_{8}+i \mathbf{x}_{8},
\end{aligned}
$$

where $\check{x}_{I}=x_{I}^{4} \frac{1}{2}, \mathbf{x}_{I}=x_{I}^{\alpha} \frac{\sigma^{\alpha}}{2}(\alpha=1,2,3)$, and $v$ is a very large vacuum expectation value of $\operatorname{Tr} X_{8}$. 
We also introduce

$$
A_{\mu}^{ \pm}=\frac{1}{2}\left(A_{\mu} \pm \hat{A}_{\mu}\right),
$$

and then $A_{\mu}^{-}$becomes an auxiliary gauge field which we can integrate out using its equation of motion. We can rewrite the covariant derivatives as

$$
D_{\mu} X_{I}=\tilde{D}_{\mu} X_{I}+i\left\{A_{\mu}^{-}, X_{I}\right\} \quad \text { with } \quad \tilde{D}_{\mu} X_{I}=\partial_{\mu} X_{I}+i\left[A_{\mu}^{+}, X_{I}\right] .
$$

In order to consider the type IIA limit for the coupling proposed in (2.9), we have to obtain the form of covariant derivatives for the transverse scalars $X_{I}$ in the limit. Taking into account the contributions from Chern-Simons term (2.6), the kinetic term for the transverse scalar fields and the WZ-type terms, we will solve the equation of motion for $A_{\mu}^{-}$in the limit of a large vacuum expectation value $v$ and large Chern-Simons level $k$, with a fixed $v / k$. It turns out that the leading term in the solution to $A_{\mu}^{-}$is linear in $1 / v$ and we can neglect every term containing $A_{\mu}^{-}$unless it is multiplied by $v$ or $k$. Keeping this in mind we rewrite the covariant derivatives for $X_{8}$ and the other transverse scalars $X_{i}$ as follows

$$
\begin{aligned}
& D_{\mu} X_{8}=\partial_{\mu} \check{x}_{8}+i v\left(A_{\mu}^{-}+\frac{1}{v} \tilde{D}_{\mu} \mathbf{x}_{8}\right), \\
& D_{\mu} X_{i}=\tilde{D}_{\mu} X_{i} .
\end{aligned}
$$

Here we notice that the appearance of $A_{\mu}^{-}$in the WZ-type action is only through $D_{\mu} X_{8}$, which means it always appears in the combination $\left(A_{\mu}^{-}+\frac{1}{v} \tilde{D}_{\mu} \mathbf{x}_{8}\right) . A_{\mu}^{-}$in the $S_{X}+S_{\mathrm{CS}}$ also appears only in this combination as verified by Ref. [7. Therefore, applying the Higgs mechanism, we make a shift of the gauge field $A_{\mu}^{-} \rightarrow A_{\mu}^{-}-\frac{1}{v} D_{\mu}^{+} x_{8}$ to eliminate the traceless part of the eighth scalar field $\mathbf{x}_{8}$ in the resulting Lagrangian. With this shift the covariant derivative of $X_{8}$ becomes

$$
D_{\mu} X_{8}=\partial_{\mu} \check{x}_{8}+i v A_{\mu}^{-} .
$$

We also adopt the Higgs rule in Ref. [8] for the covariant derivatives of the scalars $X_{i}$ and rewrite them as

$$
D_{\mu} X_{i} \rightarrow i \tilde{D}_{\mu} \tilde{X}_{i}, \quad\left(D_{\mu} X_{i}\right)^{\dagger} \rightarrow-i \tilde{D}_{\mu} \tilde{X}_{i},
$$

where $\tilde{X}_{i}=\check{x}_{i}+\mathbf{x}_{i}$ are $\mathrm{U}(2)$ adjoint scalars.

Now the action for the scalar fields (2.5) will take the following simple form

$$
S_{X}=\int d^{3} x \operatorname{Tr}\left(-\tilde{D}_{\mu} \tilde{X}_{i} \tilde{D}^{\mu} \tilde{X}_{i}-\partial_{\mu} \check{x}_{8} \partial^{\mu} \check{x}_{8}-v^{2} A_{\mu}^{-} A^{-\mu}-V_{\text {bos }}\right)+\mathcal{O}\left(\frac{1}{v}\right),
$$


where $V_{\text {bos }}$ is the potential term. This term will not be affected by the WZ-type action. Therefore, we will not write it explicitly except in the final result. The Chern-Simons action (2.6) also reduces to

$$
S_{\mathrm{CS}}=\frac{k}{2 \pi} \int d^{3} x \epsilon^{\mu \nu \rho} \operatorname{Tr}\left(A_{\mu}^{-} \tilde{F}_{\nu \rho}\right)+\mathcal{O}\left(\frac{1}{v}\right) \quad \text { with } \quad \tilde{F}_{\mu \nu}=\partial_{\mu} A_{\nu}^{+}-\partial_{\nu} A_{\mu}^{+}+i\left[A_{\mu}^{+}, A_{\nu}^{+}\right]
$$

For our immediate purpose of solving the $A_{\mu}^{-}$equation of motion, we write only the part of the WZ-type action that involves $A_{\mu}^{-}$explicitly, leaving the remaining part implicit until the end of this section

$$
\begin{aligned}
S_{C}= & \int \frac{1}{3 !} d^{3} x \epsilon^{\mu \nu \rho} \operatorname{Tr}\left\{\frac { \mu _ { 2 } } { 2 } \left[\frac{1}{2}\left(\hat{C}_{\mu \nu \rho}+C_{\mu \nu \rho}\right)-3 i \lambda C_{\mu \nu i} \tilde{D}_{\rho} \tilde{X}_{i}+3 \lambda C_{\mu \nu 8}\left(\partial_{\rho} \check{x}_{8}-i v A_{\rho}^{-}\right)\right.\right. \\
& +\frac{3}{2} \lambda^{2}\left(\hat{C}_{\mu i j}+C_{\mu i j}\right) \tilde{D}_{\mu} \tilde{X}_{i} \tilde{D}_{\nu} \tilde{X}_{j}-\frac{3}{2} i \lambda^{2} \hat{C}_{\mu i 8}\left(\tilde{D}_{\nu} \tilde{X}_{i}\left(\partial_{\rho} \check{x}_{8}+i v A_{\rho}^{-}\right)-\left(\partial_{\rho} \check{x}_{8}-i v A_{\rho}^{-}\right) \tilde{D}_{\nu} \tilde{X}_{i}\right) \\
& +\frac{3}{2} i \lambda^{2} C_{\mu i 8}\left(\tilde{D}_{\nu} \tilde{X}_{i}\left(\partial_{\rho} \check{x}_{8}-i v A_{\rho}^{-}\right)-\left(\partial_{\rho} \check{x}_{8}+i v A_{\rho}^{-}\right) \tilde{D}_{\nu} \tilde{X}_{i}\right)-i \lambda^{3} C_{i j k} \tilde{D}_{\mu} \tilde{X}_{i} \tilde{D}_{\nu} \tilde{X}_{j} \tilde{D}_{\rho} \tilde{X}_{k} \\
& +\lambda^{3} C_{i j 8}\left(\tilde{D}_{\mu} \tilde{X}_{i} \tilde{D}_{\nu} \tilde{X}_{j}\left(\partial_{\rho} \check{x}_{8}-i v A_{\rho}^{-}\right)-\tilde{D}_{\mu} \tilde{X}_{i}\left(\partial_{\rho} \check{x}_{8}+i v A_{\rho}^{-}\right) \tilde{D}_{\nu} \tilde{X}_{j}\right. \\
& \left.\left.+\left(\partial_{\rho} \check{x}_{8}-i v A_{\rho}^{-}\right) \tilde{D}_{\mu} \tilde{X}_{i} \tilde{D}_{\nu} \tilde{X}_{j}\right)\right]+(\text { c.c. }) \\
& +\frac{\mu_{2}^{\prime}}{2}\left[C_{\mu \nu \rho i j 8} X_{i j 8}^{\dagger}+\cdots+\frac{3}{2} i \lambda\left(\hat{C}_{\mu \nu i j 8 k}\left\langle\left\langle X_{i j 8}^{\dagger} \tilde{D}_{\rho} \tilde{X}_{k}\right\rangle\right\rangle-C_{\mu \nu i j 8 k}\left\langle\left\langle X_{i j 8} \tilde{D}_{\rho} \tilde{X}_{k}\right\rangle\right\rangle\right)+\cdots\right. \\
& +3 \lambda^{2} C_{\mu i j 8 k l}\left\langle\left\langle X_{i j 8}^{\dagger} \tilde{D}_{\nu} \tilde{X}_{k} \tilde{D}_{\rho} \tilde{X}_{l}\right\rangle\right\rangle+\cdots+\frac{1}{2} i \lambda^{3} \hat{C}_{i j 8 k l m}\left\langle\left\langle X_{i j 8}^{\dagger} \tilde{D}_{\mu} \tilde{X}_{k} \tilde{D}_{\nu} \tilde{X}_{l} \tilde{D}_{\rho} \tilde{X}_{m}\right\rangle\right\rangle+\cdots \\
& \left.\left.+\frac{1}{2} i \lambda^{3} C_{i j 8 k l m}\left\langle\left\langle X_{i j 8} \tilde{D}_{\mu} \tilde{X}_{k} \tilde{D}_{\nu} \tilde{X}_{l} \tilde{D}_{\rho} \tilde{X}_{m}\right\rangle\right\rangle+\cdots\right]+(\text { c.c. })\right\}+\mathcal{O}\left(\frac{1}{v}\right) .
\end{aligned}
$$

For the terms proportional to $\mu_{2}^{\prime}$ in (3.9), we kept the leading terms proportional to $v$ but neglected all the higher order terms, $\mathcal{O}\left(k / v, k / v^{2}, \ldots\right)$. The reason is that, as we pointed out before, $\mu_{2}^{\prime} \sim \beta$ and we will show shortly that the numerical factor $\beta$ is of the order of $1 / k$ which is of order of $1 / v$.

The variation of the action with respect to $A_{\mu}^{-}$gives

$$
\begin{aligned}
0=\operatorname{Tr} & \left\{\left[-2 v^{2} A^{-\mu}+\frac{k}{2 \pi} \epsilon^{\mu \nu \rho} F_{\nu \rho}+\mu_{2} v \lambda \epsilon^{\mu \nu \rho}\left[-\frac{i}{4}\left(C_{\nu \rho 8}-C_{\nu \rho 8}^{\dagger}\right)\right.\right.\right. \\
& +\frac{\lambda}{4}\left(\left(\hat{C}_{\nu i 8}+C_{\nu i 8}\right) \tilde{D}_{\rho} \tilde{X}_{i}+\tilde{D}_{\rho} \tilde{X}_{i}\left(\hat{C}_{\nu i 8}+C_{\nu i 8}\right)\right)-\frac{i \lambda^{2}}{12}\left(\left(C_{i j 8}-C_{i j 8}^{\dagger}\right) \tilde{D}_{\nu} \tilde{X}_{i} \tilde{D}_{\rho} \tilde{X}_{j}\right. \\
& \left.\left.\left.\left.+\tilde{D}_{\nu} \tilde{X}_{i}\left(C_{i j 8}-C_{i j 8}^{\dagger}\right) \tilde{D}_{\rho} \tilde{X}_{j}+\tilde{D}_{\nu} \tilde{X}_{i} \tilde{D}_{\rho} \tilde{X}_{j}\left(C_{i j 8}-C_{i j 8}^{\dagger}\right)\right)\right]\right] \delta A_{\mu}^{-}\right\} .
\end{aligned}
$$

After integrating out $A_{\mu}^{-}$, we recall that the two $\mathrm{SU}(2)$ groups will be identified and as a result $C$ and $\hat{C}$ become the same. In addition, if we identify the NS-NS two-form field in type II string 
theory as

$$
\tilde{B}_{P R}=\frac{1}{4}\left[C_{P R 8}+C_{P R 8}^{\dagger}-i\left(C_{P R 8}-C_{P R 8}^{\dagger}\right)\right]
$$

then the quantity inside square bracket in (3.10) gives the traceless part of pullback of $\tilde{B}_{\mu \nu}$,

$P\left[\tilde{B}_{\mu \nu}\right]=\tilde{B}_{\mu \nu}+\lambda \tilde{B}_{\mu i} \tilde{D}_{\nu} \tilde{X}_{i}+\lambda \tilde{D}_{\nu} \tilde{X}_{i} \tilde{B}_{\mu i}+\frac{\lambda^{2}}{3}\left(\tilde{B}_{i j} \tilde{D}_{\nu} \tilde{X}_{i} \tilde{D}_{\rho} \tilde{X}_{j}+\tilde{D}_{\nu} \tilde{X}_{i} \tilde{B}_{i j} \tilde{D}_{\rho} \tilde{X}_{j}+\tilde{D}_{\nu} \tilde{X}_{i} \tilde{D}_{\rho} \tilde{X}_{j} \tilde{B}_{i j}\right)$

On the other hand, since $\delta A_{\mu}^{-}$is traceless, the product of the trace part of $P\left[\tilde{B}_{\mu \nu}\right]$ and $\delta A_{\mu}^{-}$is also traceless. Therefore, the $A_{\mu}^{-}$equation of motion is simplified as

$$
0=\operatorname{Tr}\left\{\left[-2 v^{2} A_{\mu}^{-}+\frac{k}{2 \pi} \epsilon^{\mu \nu \rho}\left(F_{\nu \rho}+\mu_{2} v \lambda \frac{2 \pi}{k} P\left[\tilde{B}_{\nu \rho}\right]\right)\right] \delta A_{\mu}^{-}\right\} .
$$

Noting that the Yang-Mills coupling constant $g_{\mathrm{YM}}$ in the effective action of two D2-branes, the dimensionless string coupling constant $g_{\mathrm{s}}$, and the string scale $\tilde{\lambda}$ are given by

$$
g_{\mathrm{YM}}=\frac{2 \pi v}{k}, \quad g_{\mathrm{s}}=g_{\mathrm{YM}}^{2} l_{\mathrm{s}}, \quad \tilde{\lambda}=2 \pi l_{\mathrm{s}}^{2}
$$

with $\mu_{2} \lambda=\frac{1}{4 \pi^{2} l_{\mathrm{P}}^{3}} 2 \pi l_{\mathrm{P}}^{3 / 2}$ and $l_{\mathrm{P}}=g_{\mathrm{s}}^{1 / 3} l_{\mathrm{s}}, A_{\mu}^{-}$in $(\underline{3.13)})$ is

$$
A_{\mu}^{-}=\frac{1}{2 g_{\mathrm{YM}} \tilde{\lambda} v} \epsilon_{\mu}^{\nu \rho}\left(P\left[B_{\nu \rho}\right]+\tilde{\lambda} F_{\nu \rho}\right)
$$

where $B_{\mu \nu}$ is the traceless part of $\tilde{B}_{\mu \nu}$.

The gauge singlet scalar $\check{x}_{8}$ is dualized to a $\mathrm{U}(1)$ gauge field by replacing

$$
\partial_{\mu} \check{x}_{8}=\frac{1}{2 g_{\mathrm{YM}}} \epsilon_{\mu \nu \rho} \check{F}^{\nu \rho}
$$

where $\check{F}_{\nu \lambda}$ is a U(1) gauge field strength. The action for the scalar fields $S_{X}(2.5)$ is now given by

$$
S_{X}=\int d^{3} x \operatorname{Tr}\left[-\tilde{D}_{\mu} \tilde{X}_{i} \tilde{D}^{\mu} \tilde{X}_{i}-\frac{1}{2 g_{\mathrm{YM}}^{2}} \check{F}_{\mu \nu} \check{F}^{\mu \nu}+\frac{1}{2 g_{\mathrm{YM}}^{2}}\left(F_{\mu \nu}+\frac{1}{\tilde{\lambda}} P\left[B_{\mu \nu}\right]\right)^{2}-V_{\mathrm{bos}}\right]+\mathcal{O}\left(\frac{1}{v}\right),
$$

while the Chern-Simons action $(2.6)$ is given by

$$
S_{\mathrm{CS}}=\int d^{3} x \operatorname{Tr}\left[-\frac{1}{g_{\mathrm{YM}}^{2}}\left(F_{\mu \nu}+\frac{1}{\tilde{\lambda}} P\left[B_{\mu \nu}\right]\right) F^{\mu \nu}\right]+\mathcal{O}\left(\frac{1}{v}\right) .
$$


By introducing the $\mathrm{U}(2)$ adjoint antisymmetric three-form fields

$$
\tilde{C}_{P R S}=\frac{1}{2}\left[C_{P R S}+C_{P R S}^{\dagger}-i\left(C_{P R S}-C_{P R S}^{\dagger}\right)\right],
$$

we have the $C_{(3)}$ part of the WZ-type action $S_{C}$,

$$
\begin{aligned}
& S_{C}^{(3)}=\int d^{3} x \epsilon^{\mu \nu \rho} \operatorname{Tr}\left\{\frac{\mu_{2}}{3 !}\left[\tilde{C}_{\mu \nu \rho}+3 \lambda \tilde{C}_{\mu \nu i} \tilde{D}_{\rho} \tilde{X}_{i}+3 \lambda^{2} \tilde{C}_{\mu i j} \tilde{D}_{\mu} \tilde{X}_{i} \tilde{D}_{\nu} \tilde{X}_{j}+\lambda^{3} \tilde{C}_{i j k} \tilde{D}_{\mu} \tilde{X}_{i} \tilde{D}_{\nu} \tilde{X}_{j} \tilde{D}_{\rho} \tilde{X}_{k}\right]\right. \\
& \left.+\mu_{2} \lambda\left(v P\left[B_{\mu \nu}\right] A_{\rho}^{-}+P\left[\check{B}_{\mu \nu}\right] \partial_{\rho} \check{x}_{8}\right)\right\}+\mathcal{O}\left(\frac{1}{v}\right)
\end{aligned}
$$

where $P\left[\check{B}_{\mu \nu}\right]$ is the trace part $P\left[\tilde{B}_{\mu \nu}\right]$. Substituting (3.15)-(3.16) into (3.20) for $A_{\mu}^{-}$and $\partial_{\rho} \check{x}_{8}$ and taking into account the constants given in (3.14), we obtain

$$
\begin{aligned}
S_{C}^{(3)}=\int d^{3} x \operatorname{Tr}\left\{\frac{\mu_{2}}{3 !} \epsilon^{\mu \nu \rho}[\right. & \left.\tilde{C}_{\mu \nu \rho}+3 \lambda \tilde{C}_{\mu \nu i} \tilde{D}_{\rho} \tilde{X}_{i}+3 \lambda^{2} \tilde{C}_{\mu i j} \tilde{D}_{\mu} \tilde{X}_{i} \tilde{D}_{\nu} \tilde{X}_{j}+\lambda^{3} \tilde{C}_{i j k} \tilde{D}_{\mu} \tilde{X}_{i} \tilde{D}_{\nu} \tilde{X}_{j} \tilde{D}_{\rho} \tilde{X}_{k}\right] \\
& \left.-\frac{1}{g_{\mathrm{YM}}^{2} \tilde{\lambda}}\left(\lambda P\left[B_{\mu \nu}\right]\left(F^{\mu \nu}+\frac{1}{\tilde{\lambda}} P\left[B^{\mu \nu}\right]\right)+\lambda P\left[\check{B}_{\mu \nu}\right] \check{F}^{\mu \nu}\right)\right\}+\mathcal{O}\left(\frac{1}{v}\right) .
\end{aligned}
$$

In order to match the mass dimension of the ten-dimensional transverse scalar fields, we rescale the scalar fields as $\tilde{X}_{i} \rightarrow \frac{\tilde{X}_{i}}{g_{\mathrm{YM}}}$. Applying this rescaling to (3.17), (3.18), (3.21) and summing them, we get

$$
\begin{aligned}
S_{X}+S_{\mathrm{CS}}+S_{C}^{(3)}= \\
\quad \int d^{3} x \operatorname{Tr}\left\{\frac { 1 } { g _ { Y M } ^ { 2 } } \left[-\tilde{D}_{\mu} \tilde{X}_{i} \tilde{D}^{\mu} \tilde{X}_{i}-\frac{1}{2}\left(\check{F}_{\mu \nu} \check{F}^{\mu \nu}+\frac{2}{\tilde{\lambda}} \check{F}_{\mu \nu} P\left[\check{B}^{\mu \nu}\right]\right)\right.\right. \\
\left.\quad-\frac{1}{2}\left(F_{\mu \nu}+\frac{1}{\tilde{\lambda}} P\left[B_{\mu \nu}\right]\right)\left(F^{\mu \nu}+\frac{1}{\tilde{\lambda}} P\left[B^{\mu \nu}\right]\right)-\frac{1}{2}\left[\tilde{X}_{i}, \tilde{X}_{j}\right]\left[\tilde{X}_{i}, \tilde{X}_{j}\right]\right] \\
\left.+\frac{\mu_{2}}{3 !} \epsilon^{\mu \nu \rho}\left[\tilde{C}_{\mu \nu \rho}+3 \tilde{\lambda} \tilde{C}_{\mu \nu i} \tilde{D}_{\rho} \tilde{X}_{i}+3 \tilde{\lambda}^{2} \tilde{C}_{\mu i j} \tilde{D}_{\mu} \tilde{X}_{i} \tilde{D}_{\nu} \tilde{X}_{j}+\tilde{\lambda}^{3} \tilde{C}_{i j k} \tilde{D}_{\mu} \tilde{X}_{i} \tilde{D}_{\nu} \tilde{X}_{j} \tilde{D}_{\rho} \tilde{X}_{k}\right]\right\},
\end{aligned}
$$

where we used the explicit form of the scalar potential $V_{\text {bos }}$ in Ref. [7].

Next we turn to the $C_{(6)}$ part of $S_{C}$. After the aforementioned rescaling $\tilde{X}_{i} \rightarrow \frac{\tilde{X}_{i}}{g_{\mathrm{YM}}}$, the Higgs rule for $X_{i j 8}$ [8] becomes

$$
X_{i j 8} \rightarrow \frac{v}{4 g_{\mathrm{YM}}^{2}}\left[\tilde{X}_{i}, \tilde{X}_{j}\right], \quad X_{i j 8}^{\dagger} \rightarrow-\frac{v}{4 g_{\mathrm{YM}}^{2}}\left[\tilde{X}_{i}, \tilde{X}_{j}\right]
$$

We also introduce the $\mathrm{U}(2)$ adjoint antisymmetric six-form fields as

$$
\tilde{C}_{P Q R S T V}=\frac{1}{2}\left[C_{P Q R S T V}+C_{P Q R S T V}^{\dagger}-i\left(C_{P Q R S T V}-C_{P Q R S T V}^{\dagger}\right)\right]
$$


and identify the antisymmetric five-form fields in type IIA string theory as

$$
\tilde{C}_{P Q R S T}=\tilde{C}_{P Q R S T 8} .
$$

The first term in the $C_{(6)}$ Lagrangian in (3.9) is given by

$$
\frac{\mu_{2}^{\prime}}{2} \operatorname{Tr}\left(C_{\mu \nu \rho I J K} X_{I J K}^{\dagger}+C_{\mu \nu \rho I J K}^{\dagger} X_{I J K}\right)=-i \frac{3 v \mu_{2}^{\prime}}{8 g_{\mathrm{YM}}^{2}} \operatorname{Tr}\left\{-i\left(C_{\mu \nu \rho i j 8}-C_{\mu \nu \rho i j 8}^{\dagger}\right)\left[\tilde{X}_{i}, \tilde{X}_{j}\right]\right\}+\mathcal{O}\left(\frac{1}{v}\right) .
$$

Since $\left[\tilde{X}_{i}, \tilde{X}_{j}\right]$ is traceless, the product $\left(C_{\mu \nu \rho i j 8}+C_{\mu \nu \rho i j 8}^{\dagger}\right)\left[\tilde{X}_{i}, \tilde{X}_{j}\right]$ is traceless. Therefore, we can freely include this term in the last equation (3.26) to get

$$
\begin{aligned}
& \frac{\mu_{2}^{\prime}}{2} \operatorname{Tr}\left(C_{\mu \nu \rho I J K} X_{I J K}^{\dagger}+C_{\mu \nu \rho I J K}^{\dagger} X_{I J K}\right) \\
& =-i \frac{3 v \mu_{2}^{\prime}}{8 g_{\mathrm{YM}}^{2}} \operatorname{Tr}\left(\tilde{C}_{\mu \nu \rho i j 8}\left[\tilde{X}_{i}, \tilde{X}_{j}\right]\right)=-i \frac{\mu_{2} \tilde{\lambda}}{2} \operatorname{Tr}\left(\tilde{C}_{\mu \nu \rho i j}\left[\tilde{X}_{i}, \tilde{X}_{j}\right]\right) .
\end{aligned}
$$

In the second equality we have used $\mu_{2}^{\prime}=\beta \lambda \mu_{2}$ and have chosen $\beta=\frac{4 \pi}{3 k}$ in order to match the coefficient with the coefficient of the corresponding term in type IIA string theory in (2.3). Following the same procedure, we can calculate the remaining terms in the $C_{(6)}$ Lagrangian in (3.9),

$$
\begin{aligned}
& \frac{3}{2} \mu_{2}^{\prime} \lambda \operatorname{Tr}\left(C_{\mu \nu L I J K}\left\langle\left\langle X_{I J K}^{\dagger} D_{\rho} X_{L}\right\rangle\right\rangle\right)+\text { c.c. }=-\frac{3 i}{2} \mu_{2} \tilde{\lambda}^{2} \operatorname{Tr}\left(\tilde{C}_{\mu \nu i j k}\left\langle\left\langle\left[\tilde{X}_{i}, \tilde{X}_{j}\right] \tilde{D}_{\rho} \tilde{X}_{k}\right\rangle\right\rangle\right), \\
& \frac{3}{2} \mu_{2}^{\prime} \lambda^{2} \operatorname{Tr}\left(C_{\mu L M I J K}\left\langle\left\langle X_{I J K}^{\dagger} D_{\nu} X_{L}\left(D_{\rho} X_{M}\right)^{\dagger}\right\rangle\right)+\right.\text { c.c. } \\
& =-\frac{3 i}{2} \mu_{2} \tilde{\lambda}^{3} \operatorname{Tr}\left(\tilde{C}_{\mu i j k l}\left\langle\left\langle\left[\tilde{X}_{i}, \tilde{X}_{j}\right] \tilde{D}_{\nu} \tilde{X}_{k} \tilde{D}_{\rho} \tilde{X}_{l}\right\rangle\right\rangle\right), \\
& \frac{1}{2} \mu_{2}^{\prime} \lambda^{3} \operatorname{Tr}\left(C_{L M N I J K}\left\langle\left\langle X_{I J K}^{\dagger} D_{\mu} X_{L}\left(D_{\nu} X_{M}\right)^{\dagger} D_{\rho} X_{N}\right\rangle\right)+\right.\text { c.c. } \\
& =-\frac{i}{2} \mu_{2} \tilde{\lambda}^{4} \operatorname{Tr}\left(\tilde{C}_{i j k l m}\left\langle\left\langle\left[\tilde{X}_{i}, \tilde{X}_{j}\right] \tilde{D}_{\mu} \tilde{X}_{k} \tilde{D}_{\nu} \tilde{X}_{l} \tilde{D}_{\rho} \tilde{X}_{m}\right\rangle\right\rangle\right) .
\end{aligned}
$$

Summing (3.22) and (3.27)-(3.30), we finally reach

$$
\begin{aligned}
S_{\text {tot }}= & \int d^{3} x \operatorname{Tr}\left\{\frac { 1 } { g _ { Y M } ^ { 2 } } \left[-\tilde{D}_{\mu} \tilde{X}_{i} \tilde{D}^{\mu} \tilde{X}_{i}-\frac{1}{2}\left(\check{F}_{\mu \nu} \check{F}^{\mu \nu}+\frac{2}{\tilde{\lambda}} \check{F}_{\mu \nu} P\left[\check{B}^{\mu \nu}\right]\right)\right.\right. \\
& \left.-\frac{1}{2}\left(F_{\mu \nu}+\frac{1}{\tilde{\lambda}} P\left[B_{\mu \nu}\right]\right)\left(F^{\mu \nu}+\frac{1}{\tilde{\lambda}} P\left[B^{\mu \nu}\right]\right)-\frac{1}{2}\left[\tilde{X}_{i}, \tilde{X}_{j}\right]\left[\tilde{X}_{i}, \tilde{X}_{j}\right]\right] \\
& +\frac{\mu_{2}}{3 !} \epsilon^{\mu \nu \rho}\left[\tilde{C}_{\mu \nu \rho}+3 \tilde{\lambda} \tilde{C}_{\mu \nu i} \tilde{D}_{\rho} \tilde{X}_{i}+3 \tilde{\lambda}^{2} \tilde{C}_{\mu i j} \tilde{D}_{\mu} \tilde{X}_{i} \tilde{D}_{\nu} \tilde{X}_{j}+\tilde{\lambda}^{3} \tilde{C}_{i j k} \tilde{D}_{\mu} \tilde{X}_{i} \tilde{D}_{\nu} \tilde{X}_{j} \tilde{D}_{\rho} \tilde{X}_{k}\right] \\
& +\frac{\mu_{2}}{3 !} \epsilon^{\mu \nu \rho}\left[-\frac{i}{2} \tilde{\lambda} \tilde{C}_{\mu \nu \rho i j 8}\left[\tilde{X}_{i}, \tilde{X}_{j}\right]-\frac{3 i}{2} \tilde{\lambda}^{2} \tilde{C}_{\mu \nu i j k}\left\langle\left\langle\left[\tilde{X}_{i}, \tilde{X}_{j}\right] \tilde{D}_{\rho} \tilde{X}_{k}\right\rangle\right\rangle\right. \\
& \left.\left.-\frac{3 i}{2} \tilde{\lambda}^{3} \tilde{C}_{\mu i j k l}\left\langle\left\langle\left[\tilde{X}_{i}, \tilde{X}_{j}\right] \tilde{D}_{\nu} \tilde{X}_{k} \tilde{D}_{\rho} \tilde{X}_{l}\right\rangle\right\rangle-\frac{i}{2} \tilde{\lambda}^{4} \tilde{C}_{i j k l m}\left\langle\left\langle\tilde{X}_{i}, \tilde{X}_{j}\right] \tilde{D}_{\mu} \tilde{X}_{k} \tilde{D}_{\nu} \tilde{X}_{l} \tilde{D}_{\rho} \tilde{X}_{m}\right\rangle\right]\right\} .
\end{aligned}
$$


The second and third terms in (3.31) are unified to form the kinetic term of $\mathrm{U}(2)$ gauge field, as the gauge invariant combination on the world-volume of D-brane,

$$
-\frac{1}{2 g_{\mathrm{YM}}^{2}} \operatorname{Tr}\left[\left(\tilde{F}_{\mu \nu}+\frac{1}{\tilde{\lambda}} P\left[\tilde{B}_{\mu \nu}\right]\right)\left(\tilde{F}^{\mu \nu}+\frac{1}{\tilde{\lambda}} P\left[\tilde{B}^{\mu \nu}\right]\right)\right]
$$

up to the quadratic term in $P\left[\check{B}_{\mu \nu}\right]$ which belongs to nonlinear terms in $C^{(3)}$. Here we notice that in addition to the natural couplings of the D2-brane to the three-form field and the dual fiveform field in type IIA superstring theory, the WZ-type action also produces the coupling between $\tilde{F}_{\mu \nu}$ and $\tilde{B}_{\mu \nu}$ in the linearized nonabelian DBI action for D2-brane. Unfortunately, however, the dimensional reduction of the WZ-type action (2.8) does not produce $\tilde{C}^{(3)} \wedge \tilde{F}$ and $\tilde{C}^{(5)} \wedge \tilde{F}$-terms which appear in ten dimensional WZ-type action (2.3). We need more investigations in this direction.

Our derivation of the result in (3.31) is based on the elegant Higgs rule of [8]. Here we would like to comment on a mild problem in applying these rules. We know that the transverse scalars $X_{i}$ are bi-fundamentals of $\mathrm{SU}(2) \times \mathrm{SU}(2)$. Therefore, to obtain the $\mathrm{U}(2)$ adjoint scalar, the trace and the traceless part of these scalars should be combined as $\tilde{X}_{i}=\check{x}_{i}+\mathbf{x}_{i}$. A similar rewriting should also be made for the form fields. When we are dealing with the BLG theory without WZtype coupling, the Higgs rule of [8] are exactly the net effect of this splitting and recombination of the trace and traceless part of the fields. However, in the presence of the WZ-type coupling containing more than two covariant derivatives, the splitting and recombination of the trace and traceless parts reexpress most of the terms of $S_{C}$ in terms of the $\mathrm{U}(2)$ adjoint fields except a few terms which lead to some mismatch. To demonstrate this observation we consider the $C_{i j k}$-term,

$$
\begin{aligned}
& \frac{1}{2} \epsilon^{\mu \nu \rho} \operatorname{Tr}\left[C_{i j k}\left(\tilde{D}_{\mu} X_{i}\right)^{\dagger} \tilde{D}_{\nu} X_{j}\left(\tilde{D}_{\rho} X_{k}\right)^{\dagger}+C_{i j k}^{\dagger} \tilde{D}_{\mu} X_{i}\left(\tilde{D}_{\nu} X_{j}\right)^{\dagger} \tilde{D}_{\rho} X_{k}\right] \\
& =\epsilon^{\mu \nu \rho} \operatorname{Tr}\left(\tilde{C}_{i j k} \tilde{D}_{\mu} \tilde{X}_{i} \tilde{D}_{\nu} \tilde{X}_{j} \tilde{D}_{\rho} \tilde{X}_{k}-2 \tilde{C}_{i j k} \tilde{D}_{\mu} \check{x}_{i} \tilde{D}_{\nu} \check{x}_{j} \tilde{D}_{\rho} x_{k}-2 \tilde{C}_{i j k} \tilde{D}_{\mu} \check{x}_{i} \tilde{D}_{\nu} x_{j} \tilde{D}_{\rho} x_{k}\right)
\end{aligned}
$$

where the covariant derivative is given in (3.3). We have also made the following splitting and recombination of the trace and traceless part of the three-form field

$$
C_{i j k}=\frac{1}{2} \tilde{c}_{i j k} \mathbf{1}+i c_{i j k}^{\alpha} \frac{\sigma^{\alpha}}{2}, \quad C_{i j k}^{\dagger}=\frac{1}{2} \tilde{c}_{i j k} \mathbf{1}-i c_{i j k}^{\alpha} \frac{\sigma^{\alpha}}{2}, \quad \tilde{C}_{i j k}=\frac{1}{2} \tilde{c}_{i j k} \mathbf{1}+c_{i j k}^{\alpha} \frac{\sigma^{\alpha}}{2} .
$$

Note that the the last two terms in (3.33) cannot entirely be expressed in terms of the U(2) adjoint fields. This mismatch is generated from the cross terms between the trace and traceless sectors. It is quite straightforward to show that, in the $\mathrm{U}(1) \times \mathrm{U}(1)$ ABJM theory, there is no such mismatch. We will leave verification of the absence of such mismatch in ABJM theory with arbitrary gauge group for the future work [19]. 


\section{Quartic Mass-Deformation Term from a $C_{(6)}$ Term}

Let us recall the bosonic part of supersymmetry-preserving mass-deformation terms in the BLG theory [13, 14] in order to compare these with the WZ-type action in (2.9),

$$
\begin{array}{r}
S_{m}=\int d^{3} x m^{2} \operatorname{Tr}\left(X_{I} X_{I}^{\dagger}+X_{I}^{\dagger} X_{I}\right)+\frac{4 \pi m}{k} \int d^{3} x \operatorname{Tr}\left[X_{3}\left(X_{4}\right)^{\dagger} X_{5}\left(X_{6}\right)^{\dagger}-X_{5}\left(X_{4}\right)^{\dagger} X_{3}\left(X_{6}\right)^{\dagger}\right. \\
\left.+X_{7}\left(X_{8}\right)^{\dagger} X_{9}\left(X_{10}\right)^{\dagger}-X_{9}\left(X_{8}\right)^{\dagger} X_{7}\left(X_{10}\right)^{\dagger}\right],
\end{array}
$$

where $m$ is the mass parameter. According to [12], this mass term comes from the background fourform flux which is (anti-)self-dual in eight-dimensional transverse space. We examine specifically how (4.1) can appear from WZ-type coupling (2.8). Due to the (anti-)self-dual property of the flux, we should consider the contribution from both four-form $F_{(4)}$ and dual seven-form $F_{(7)}$. Let us first take into account the contribution from $F_{(7)}$ by turning on only specific components of $F_{(7)}$ as

$$
F_{\mu \nu \rho I J K L}=\frac{\beta m}{\lambda \mu_{2}^{\prime}} \epsilon_{\mu \nu \rho} T_{I J K L},
$$

where $T_{I J K L}$ is (anti-)self-dual in eight-dimensional transverse space

$$
T^{I J K L}= \pm \frac{1}{4 !} \epsilon^{I J K L I^{\prime} J^{\prime} K^{\prime} L^{\prime}} T_{I^{\prime} J^{\prime} K^{\prime} L^{\prime}}
$$

The corresponding WZ-type action of our consideration (2.9) is

$$
\left.S_{C}^{(6)}=\int d x^{\mu} \wedge d x^{\nu} \wedge d x^{\rho} \frac{\mu_{2}^{\prime}}{2} \operatorname{Tr}\left[C_{\mu \nu \rho I J K}\left(X_{I}\right)^{\dagger} X_{J}\left(X_{K}\right)^{\dagger}+\text { (c.c. }\right)\right]+\cdots
$$

When the six-form $C_{\mu \nu \rho I J K}$ is the potential of constant seven-form field strength $F_{\mu \nu \rho I J K L}$, we obtain it explicitly,

$$
C_{\mu \nu \rho I J K}=\lambda F_{\mu \nu \rho I J K L} X_{L}=\frac{\beta m}{\mu_{2}^{\prime}} \epsilon_{\mu \nu \rho} T_{I J K L} X_{L} .
$$

Substituting the six-form field configuration (4.5) with (4.3) into the WZ-type action (4.4), we have

$$
\begin{aligned}
S_{C}^{(6)} & \left.=\frac{4 \pi m}{3 k} \int d x^{\mu} \wedge d x^{\nu} \wedge d x^{\rho} \epsilon_{\mu \nu \rho} \frac{1}{2} \operatorname{Tr}\left[T_{I J K L} X_{L}\left(X_{I}\right)^{\dagger} X_{J}\left(X_{K}\right)^{\dagger}+\text { (c.c. }\right)\right]+\cdots \\
& \left.=-\frac{4 \pi m}{k} \int d^{3} x \operatorname{Tr}\left[T_{I J K L} X_{L}\left(X_{I}\right)^{\dagger} X_{J}\left(X_{K}\right)^{\dagger}+\text { (c.c. }\right)\right]+\cdots
\end{aligned}
$$

which exactly coincides with the quartic mass-deformation term in (4.1) as far as the four-form tensor $T_{I J K L}$ satisfies

$$
T_{1234}=T_{5678}=1, \quad \text { other independent components }=0 .
$$


This configuration keeps the maximal supersymmetry $\mathcal{N}=8$, and turning on other components of $T_{I J K L}$ in addition to (4.7) leads to less supersymmetry. The relation between nonzero components of $T_{I J K L}$ and the number of supersymmetry has been studied in the context of field theory [14, 20, 21] and in the dual AdS side [12].

The contribution from the four-form tensor is also calculated in a similar way. From (4.2) the configuration of $F_{(4)}$ is

$$
F_{I J K L} \sim \frac{\beta m}{\lambda \mu_{2}^{\prime}} T_{I J K L}=\frac{m}{\lambda^{2} \mu_{2}} T_{I J K L} .
$$

Then the corresponding WZ-type action is

$$
\begin{aligned}
S_{C}^{(3)} & \sim \mu_{2} \int d x^{\mu} \wedge d x^{\nu} \wedge d x^{\rho} \operatorname{Tr}\left[\frac{m}{\lambda^{2} \mu_{2}} T_{I J K L} \lambda^{4} X_{L}\left(D_{\mu} X_{I}\right)^{\dagger} D_{\nu} X_{J}\left(D_{\rho} X_{K}\right)^{\dagger}+(\text { c.c. })\right]+\cdots \\
& =\lambda^{2} m \int d x^{\mu} \wedge d x^{\nu} \wedge d x^{\rho} \operatorname{Tr}\left[T_{I J K L} X_{L}\left(D_{\mu} X_{I}\right)^{\dagger} D_{\nu} X_{J}\left(D_{\rho} X_{K}\right)^{\dagger}+(\text { c.c. })\right]+\cdots
\end{aligned}
$$

Since $\lambda^{2} \sim l_{\mathrm{P}}^{3}$, this term does not contribute under the limit $l_{\mathrm{P}} \rightarrow 0$.

We confirm the identification of a cubic WZ-type term (4.4) with a specific form of constant (anti-)self-dual four-form flux (4.2) in Minkowski spacetime signature. If we take a Euclideanization to the flux (4.2), then an overall imaginary number $i$ appears in the left-hand side. It implies that $\mu$ in (4.2) may not be a mass parameter but a chemical potential.

For the quadratic mass term which can be interpreted as the quadratic coupling of form fields between M2's, we do not have natural argument to fix it. In the case of string theory, this coupling is obtained from the world-sheet disk amplitude with insertion of the two R-R vertex operators. Though we basically have ambiguity for the position of two insertions, we can avoid this ambiguity by introducing appropriate auxiliary fields [22, 23] and compute the coupling at least for some particular cases [24, 25].

\section{Conclusion and Discussion}

Once the world-volume action of $N$ stacked M2-branes is determined, it is interesting to understand how the M2-branes couple to the bulk fields. In this paper, we constructed the WZ-type action which describes the coupling of the M2-branes to antisymmetric three- and six-form fields in Mtheory. We consider the BLG theory for two M2-branes and write down a WZ-type action linear to antisymmetric three- and six-form fields in analogy with the corresponding action in type IIA string theory. When it reduces to ten dimensions through a circle compactification, our action reproduces the expected ten-dimensional coupling of R-R and NS-NS form fields to D2-branes in type IIB string theory. 
In addition to our main goal of obtaining the WZ-type coupling of M2-branes, we show that a particular cubic WZ-type term can be identified with the quartic scalar interaction in the supersymmetry-preserving mass deformation of the BLG theory. We made this identification in a flat world-volume and transverse space by making an assumption that the seven-form field strength is constant and is proportional to the mass parameter of mass deformation term.

A few discussions are in order. Though the ten-dimensional WZ-type action (2.1) is restricted to the terms linear in $\tilde{C}_{(n)}$, it contains $\tilde{C}_{(n)} \wedge e^{\tilde{B}}$ where $\tilde{B}$ is NS-NS two-form field. Since the NS-NS two-form field in string theory comes from a part of $C_{(3)}$ in M-theory, inclusion of quadratic or higher order terms in $C_{(3)}$ in addition to the WZ-type action (2.9) seems natural. For instance the quadratic term in $C_{(3)}$ is

$$
\begin{aligned}
& S_{C^{2}}=\frac{\mu_{2}^{\prime \prime}}{2} \int_{2+1} \operatorname{Tr}\left(P\left[\left\langle\mathrm{i}_{X} \mathrm{i}_{X} \mathrm{i}_{X}\right\rangle C_{(3)} \wedge C_{(3)}\right]\right) \\
&=5 \mu_{2}^{\prime \prime} \int \frac{1}{3 !} d^{3} x \epsilon^{\mu \nu \rho} \operatorname{Tr}\left[\frac{1}{2} \hat{C}_{[\mu \nu \rho}\left\langle\left\langle C_{I J K]}^{\dagger} X_{I J K}\right\rangle\right\rangle+\frac{1}{2} C_{[\mu \nu \rho}\left\langle\left\langle C_{I J K]} X_{I J K}^{\dagger}\right\rangle\right\rangle\right. \\
&+ 3 \lambda C_{[\mu \nu L}\left\langle\left\langle C_{I J K]}^{\dagger} X_{I J K}\left(D_{\rho} X_{L}\right)^{\dagger}\right\rangle\right\rangle \\
&+\frac{3}{2} \lambda^{2} \hat{C}_{[\mu L M}\left\langle\left\langle C_{I J K]}^{\dagger} X_{I J K}\left(D_{\nu} X_{L}\right)^{\dagger} D_{\rho} X_{M}\right\rangle\right\rangle \\
&+\frac{3}{2} \lambda^{2} C_{[\mu L M}\left\langle\left\langle C_{I J K]} X_{I J K}^{\dagger} D_{\nu} X_{L}\left(D_{\rho} X_{M}\right)^{\dagger}\right\rangle\right\rangle \\
&\left.+\lambda^{3} C_{[L M N}\left\langle\left\langle C_{I J K]}^{\dagger} X_{I J K}\left(D_{\mu} X_{L}\right)^{\dagger} D_{\nu} X_{M}\left(D_{\rho} X_{N}\right)^{\dagger}\right\rangle\right\rangle+(\text { c.c. })\right],
\end{aligned}
$$

where $\mu_{2}^{\prime \prime}=\beta^{\prime} \lambda \mu_{2}$. Like $\beta$, the value of $\beta^{\prime}$ is also determined by comparing this term with an appropriate term in the corresponding action of type IIA superstring theory, after a circle compactification. Specifically, when $\beta^{\prime}=\beta=4 \pi / 3 k$, this term exactly coincides with the $C_{(3)} \wedge B$ term in (2.1).

In this paper we constructed the bosonic sector of WZ-type action coupled to the worldvolume fields of M2-branes. If we supersymmetrize what we obtained, then we may reach the supersymmetric WZ-type action in M-theory. Though we fixed the coefficient of the WZ-type action (2.9) by comparing with the terms of the ten-dimensional R-R coupling action (2.1) through the compactification of the eighth transverse direction, this indirect fixation procedure can be reconfirmed by constructing the supersymmetric WZ-type action coupled to M2-branes. Then, this understanding will also help the extension to general case of arbitrary number of stacked multiple M2-branes of which the world-volume theory is described by the $\mathcal{N}=6$ superconformal Chern-Simons gauge theory with $\mathrm{U}(N) \times \mathrm{U}(N)$ gauge symmetry. We will report the construction of WZ-type coupling in the context of ABJM theory for arbitrary number of M2-branes in the subsequent work [19]. 
In the BLG and ABJM theories, M2-branes and $\mathrm{M} 2$-branes are not distinguished as the case of DBI type world-volume action of D-branes. The D- and D-branes carry opposite sign R-R charges and are distinguished by the R-R coupling (2.1) in type II string theories [3]. Similarly the M2- and M2-branes are also distinguishable by the analogue of WZ-type in the M-theory (2.9)). This will also let the construction of world-volume action of M2 $\overline{\mathrm{M}} 2$ pair without supersymmetry tractable.

\section{Acknowledgements}

The authors would like to appreciate the informative discussions with Min-Young Choi and Akira Ishida. This work was supported by the Korea Research Foundation Grant funded by the Korean Government with grant number KRF-2008-313-C00170 (Y.K.), 2009-0073775 (O.K.), and 20090077423 (D.T.). This work was also supported by Astrophysical Research Center for the Structure and Evolution of the Cosmos (ARCSEC)).

\section{References}

[1] M. Li, "Boundary States of D-Branes and Dy-Strings," Nucl. Phys. B 460, 351 (1996) arXiv:hep-th/9510161.

[2] M. R. Douglas, "Branes within branes," arXiv:hep-th/9512077;

M. B. Green, J. A. Harvey and G. W. Moore, "I-brane inflow and anomalous couplings on D-branes," Class. Quant. Grav. 14, 47 (1997) arXiv:hep-th/9605033].

[3] R. C. Myers, "Dielectric-branes," JHEP 9912, 022 (1999) arXiv:hep-th/9910053].

[4] J. Bagger and N. Lambert, "Modeling multiple M2's," Phys. Rev. D 75, 045020 (2007) arXiv:hep-th/0611108]; "Gauge Symmetry and Supersymmetry of Multiple M2-Branes," Phys. Rev. D 77, 065008 (2008) arXiv:0711.0955 [hep-th]]; "Comments On Multiple M2branes," JHEP 0802, 105 (2008) arXiv:0712.3738 [hep-th]].

[5] A. Gustavsson, "Algebraic structures on parallel M2-branes," Nucl. Phys. B 811, 66 (2009) arXiv:0709.1260 [hep-th]].

[6] O. Aharony, O. Bergman, D. L. Jafferis and J. Maldacena, "N=6 superconformal ChernSimons-matter theories, M2-branes and their gravity duals," JHEP 0810, 091 (2008) arXiv:0806.1218 [hep-th]]. 
[7] S. Mukhi and C. Papageorgakis, "M2 to D2," JHEP 0805, 085 (2008) arXiv:0803.3218 [hep-th]].

[8] B. Ezhuthachan, S. Mukhi and C. Papageorgakis, "The Power of the Higgs Mechanism: Higher-Derivative BLG Theories," JHEP 0904, 101 (2009) [arXiv:0903.0003 [hep-th]].

[9] M. Li and T. Wang, "M2-branes Coupled to Antisymmetric Fluxes," JHEP 0807, 093 (2008) arXiv:0805.3427 [hep-th]].

[10] M. A. Ganjali, "On Dielectric Membranes," arXiv:0901.2642 [hep-th].

[11] J. Polchinski and M. J. Strassler, "The string dual of a confining four-dimensional gauge theory," arXiv:hep-th/0003136.

[12] I. Bena, "The M-theory dual of a 3 dimensional theory with reduced supersymmetry," Phys. Rev. D 62 (2000) 126006 arXiv:hep-th/0004142].

[13] J. Gomis, A. J. Salim and F. Passerini, "Matrix Theory of Type IIB Plane Wave from Membranes," JHEP 0808 (2008) 002 [arXiv:0804.2186 [hep-th]].

[14] K. Hosomichi, K. M. Lee and S. Lee, "Mass-Deformed Bagger-Lambert Theory and its BPS Objects," Phys. Rev. D 78, 066015 (2008) arXiv:0804.2519 [hep-th]].

[15] For a review, see P. K. Townsend, "Four lectures on M-theory," arXiv:hep-th/9612121.

[16] M. Van Raamsdonk, "Comments on the Bagger-Lambert theory and multiple M2-branes," JHEP 0805, 105 (2008) arXiv:0803.3803 [hep-th]].

[17] M. Benna, I. Klebanov, T. Klose and M. Smedback, "Superconformal Chern-Simons Theories and $\mathrm{AdS}_{4} / \mathrm{CFT}_{3}$ Correspondence," JHEP 0809, 072 (2008) [arXiv:0806.1519 [hep-th]].

[18] E. Bergshoeff and M. De Roo, "D-branes and T-duality," Phys. Lett. B 380, 265 (1996) arXiv:hep-th/9603123 ;

E. Alvarez, J. L. F. Barbon and J. Borlaf, "T-duality for open strings," Nucl. Phys. B 479, 218 (1996) arXiv:hep-th/9603089.

[19] Y. Kim, O-K. Kwon, H. Nakajima, and D. D. Tolla, in preparation.

[20] C. Ahn, "Towards Holographic Gravity Dual of N=1Superconformal Chern-Simons Gauge Theory," JHEP 0807 (2008) 101 arXiv:0806.4807 [hep-th]]; "Comments on Holographic Gravity Dual of N=6 Superconformal Chern-Simons Gauge Theory," arXiv:0812.4363 [hepth]. 
[21] H. Nishino and S. Rajpoot, "Mass Deformation Of Bagger-Lambert Theory In D-3 With Reduced N=1 Supersymmetry," Phys. Rev. D 78 (2008) 085006.

[22] J. J. Atick, L. J. Dixon and A. Sen, "String Calculation Of Fayet-Iliopoulos D Terms In Arbitrary Supersymmetric Compactifications," Nucl. Phys. B 292 (1987) 109.

[23] M. Dine, I. Ichinose and N. Seiberg, "F Terms And D Terms In String Theory," Nucl. Phys. B 293 (1987) 253.

[24] M. Billo, M. Frau, I. Pesando and A. Lerda, "N = 1/2 gauge theory and its instanton moduli space from open strings in R-R background," JHEP 0405 (2004) 023 arXiv:hep-th/0402160.

[25] K. Ito and S. Sasaki, "Non(anti)commutative $\mathrm{N}=2$ supersymmetric gauge theory from superstrings in the graviphoton background," JHEP 0611 (2006) 004 arXiv:hep-th/0608143]; K. Ito, H. Nakajima and S. Sasaki, "Deformation of Super Yang-Mills Theories in R-R 3-form Background," JHEP 0707, 068 (2007) arXiv:0705.3532 [hep-th]]. 